\title{
Current and future methodology for quantitation and site-spe- cific mapping the location of DNA adducts
}

\author{
Gunnar Boysen ${ }^{1,2}, *$ Intawat Nookaew ${ }^{2,3}$ \\ 1 Dept. Environmental and Occupational Health, Fay W. Boozman College of Public Health, University of \\ Arkansas for Medical Sciences \\ 2 The Winthrop P Rockefeller Cancer institute, University of Arkansas for Medical Sciences \\ 3 Dept. Biomedical Informatics, College of Medicine, University of Arkansas for Medical Sciences \\ * Correspondence: egboysen@uams.edu
}

\begin{abstract}
Formation of DNA adducts is a key event for a genotoxic mode of action and their formation is often used as surrogate for mutation and cancer. Interest in DNA adducts are twofold, first, to demonstrate exposure, and second, to link DNA adduct location to subsequent mutations or altered gene regulation. Methods have been established to quantify DNA adducts with high chemical specificity, and to visualize the location of DNA adducts, elegant bio-analytical methods have been devised utilizing enzymes, various chemistries, and molecular biology methods. Traditionally, these highly specific methods cannot be combined, and the results are incomparable. Initially developed for single-molecule DNA sequencing, nanopore-type technologies are expected to enable simultaneous quantitation and location of DNA adducts across the genome. Herein, we will briefly summarize the current methodologies for state-of-the-art quantitation of DNA adduct levels and mapping of DNA adducts and describe novel single-molecule DNA sequencing technologies that are expected to achieve both measures simultaneously. Emerging technologies are expected to soon provide a comprehensive picture of the exposome and identify gene regions susceptible to DNA adduct formation.
\end{abstract}

Keywords: DNA adducts; nanopore; Oxford Nanopore Technology; mass spectrometry; adductomics; exposome

\section{Introduction}

Chemical carcinogenesis. Although DNA is a very stable molecule for storing biological information, it is under relentless attack by reactive compounds of endogenous and exogenous origin that covalently bind to DNA, forming so called DNA adducts [1]. Since identification of the first DNA adduct by Reiner, B. and Zamenhof in 1957 [2], several thousand studies on DNA adducts have been reported and are reviewed from different perspectives [3-10]. The ability of a compound to form DNA adducts, directly or after metabolic activation, is considered a critical event in chemical carcinogenesis [11] and a key event for genotoxic mode of action of toxicants [12,13]. The binding to DNA, has been widely used as biomarker of exposure in molecular epidemiology studies to link exposure to adverse health outcomes [14-16]. Formation and stability of specific promutagenic DNA adducts has been established in vitro, in cell cultures, animal experiments and molecular epidemiology studies $[5,6,17]$. Recent advances in technology, especially in mass spectrometry and single molecule sequencing, allows for 'omics' type monitoring of all DNA adducts, DNA adductomics, and is expected to provide unprecedented insight into the total exposome [18-21].

This perspective summarizes the current methodologies for quantitation of DNA adduct levels and mapping the location of DNA adducts along the genome and highlight novel single-molecule analysis expected to be capable for achieving both measures. 


\section{Methodology}

Historically studies on DNA adducts have mirrored advances in analytical and bioanalytical chemistry technologies, starting with paper chromatography, enzyme-linked immunosorbent assay, ${ }^{32}$ P-Post-labeling [22], followed by liquid [23] and gas chromatography separations with various detection systems, including UV, fluorescence and electrochemical detectors $[24,25]$.

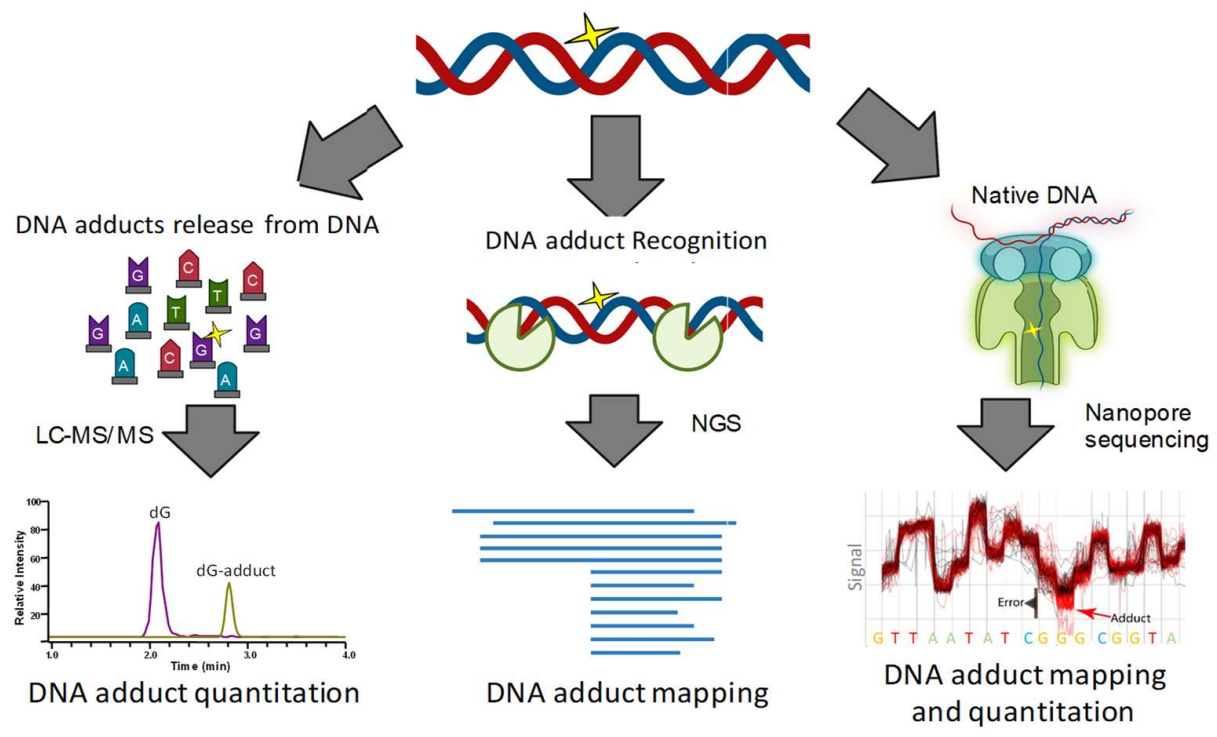

Scheme 1. Overview of current approaches for measuring DNA adduct levels and mapping the location of DNA adducts. Left: DNA digestion or release of DNA adducts, prior to quantitation by LC-MS. Middle: Labeling of DNA adduct sites prior to localizing by amplification-base sequencing. Right: Single molecule sequencing using nanopore-based technologies.

\subsection{Mass Spectrometry-based DNA Adduct quantitation.}

The introduction of electrospray ionization by Dole and others, in 1968 [26] revolutionized mass-spectrometry and biochemistry [27]. Electrospray mass-spectrometry is now a commonly used technique for qualitative and quantitative analyses of any type of compound, including DNA adducts [24,25]. Improvements of mass analyzers allows monitoring of thousands of molecules simultaneously with ultra-high mass resolution and accuracy [28-31]. The basic and most frequently used approach for quantitation of DNA adducts is to isolate DNA, liberate the DNA adducts from DNA by chemical means or enzyme hydrolysis and quantify the released nucleotide- or base-adducts by LC-MS (Scheme 1 left) [32]. To improve sensitivity various sample enrichment procedures maybe included such as solid phase and liquid-liquid extraction or pre-separation by HPLC [3]. If needed, stable isotope standards are added at the beginning to account for any potential loss during sample workup and to improve measurement accuracy [33,34].

\subsection{Adductomics, nontargeted screening of DNA adducts.}

While most studies apply targeted mass spectrometry using authentic DNA adduct standards, efforts are underway to move towards nontargeted 'omics' type screening of DNA adducts to obtain a complete measure of the exosome [35]. Therefore, DNA adducts are monitored in various modes of data dependent (MS/MS) or multistage (MSn) scanning modes $[19,20,36]$.

Independent of targeted or nontargeted approach, results are reported as number or concentration of DNA adduct per DNA, or the corresponding unmodified nucleotides (e.g. fmol/mg DNA, fmol / $\mu \mathrm{mol} \mathrm{dG).} \mathrm{The} \mathrm{mass} \mathrm{spectrometry-base} \mathrm{detection} \mathrm{provides} \mathrm{a}$ high level of chemical specificity but no site-specificity. 


\subsection{Amplification-based Mapping of DNA Adducts.}

Various methods have been developed for genome-wide and site-specific mapping of DNA damage (Scheme 1 middle). The general approach is based on mapping sequencing stop sites to localize the adducts [37-39]. Therefore, DNA adducts are first recognized or modified by enzymatic or chemical means, taking advantage of DNA repair enzymes to mark and excise DNA adducts.

The marked or cleaved DNA is then amplified, sequenced and the location of DNA adducts are obtained from strand ends or mismatched base pairs [37-39]. For example, Denissenko et al. used UvrABC excision nuclease, in combination with ligation-mediated polymerase chain reaction (PCR), to map the sites of bulky DNA adducts [40-42]. More recently, cyclobutene pyrimidine dimers (CPDs) were mapped with CPD-seq based on DNA cleavage mediated by T4 endonuclease V and APE1 [43,44]. XR-seq [45] and tXRseq rely on TFIIH-mediated enrichment of damage-containing fragments cleaved by mammalian nucleotide excision repair enzymes [46-48]. Cisplatin-seq takes advantage of the HMG box A of HMGB1 protein's preferentially binding to distorted DNA structures for selective enrichment of cisplatin-modified DNA [49]. Further, click chemistry, ClickCode-Seq, has been successfully applied to label 8-oxo-dG or 5-hm-dC sites in DNA prior to next generation sequencing $[50,51]$. DNA adduct mapping methods have been reviewed extensively [52-54].

Results are given as modified versus unmodified sites or motifs with high level of site-specificity. Unfortunately, these elegant methods are limited by (i) the breadth of enzyme specificity, which may identify a mixture of DNA adducts; (ii) excision of short DNA fragments that sometimes cannot be aligned with absolute certainty; (iii) reliance on completion of chemical reactions; (iv) restricted applicability to one type or class of DNA adducts; (v) the inability to distinguish different DNA adducts; and (v) limited chemical specificity.

\subsection{Single Molecule DNA sequencing.}

A relatively new method for analysis of DNA adducts is based on single molecule sequencing. This novel technology sequences and counts single DNA molecules, whether they are whole genomes or DNA fragments.

\subsubsection{Nanopore Technology.}

Nanopore-type technology utilizes electrochemical forces to pull single-stranded DNA in native form through tiny pores (Figure 1 right). The accompanying changes in electric current indicate the physicochemical properties of the DNA bases transiting through the pore, revealing the DNA sequence and potential DNA adducts [55]. A DNA adduct modulates the nanopore ion current signal while entering, passing through, and exiting the nanopore. This results in an electric current signature characteristic for the DNA adduct within a given 7-base sequence that includes the DNA adduct and the three adjacent $3^{\prime}$ and $5^{\prime}$ nucleotides that reside in the nanopore [55].

Burrows and colleagues pioneered the application of nanopore-type technology for sequencing DNA adducts in single-stranded DNA. With custom-made solid-state or protein-based nanopores, they showed the proof-of-principle for detecting $\mathrm{N}^{2}$-BPDE-dG-induced adducts [56], abasic sites [57-59], and other DNA adducts [60], including 8-oxo-dG [61-63].

\subsubsection{Oxford Nanopore Technologies (ONT)}

Using a similar principle, ONT developed and commercialized a nucleic acid sequencing technology that has the capability to sequence long to ultra-long molecules of DNA $(>2 \mathrm{Mb})$ in the native form, preserving the sequence position of the DNA adducts [64-66].

2.3.1.2 PacBio DNA sequencer. 
While technical not a nanopore system, the PacBio RSII DNA sequencer also employs the single-molecule real-time (SMRT) sequencing principle. The DNA strands are converted into loops and amplified by polymers. The unique time needed by the polymerase for elongation of the DNA is indicative of the base added and DNA sequence.

While ONT uses the disturbance in the ion signals, the PacBio system makes use of the time delay of the polymerase to identify DNA sequence. The standard base calling algorithms for both systems are optimized for the four main bases (A, T, G and C), and report errors when encountering unknown bases, potential DNA adducts. Therefore, efforts are under way to expand base calling algorithms to enable detection of DNA adducts and epigenetic modifications. Multiple groups successfully applied ONT for genome wide detection of epigenetic modifications, such as 5-methyl-2'-deoxycytidine (5mdC) and $N^{6}$-methyl-2'-deoxyadenosine ( $N^{6} \mathrm{mdA}$ ) [67-70]. Similarly, the PacBio system has been shown to be suitable for simultaneous detection of $N^{6} \mathrm{mdA}, 5 \mathrm{mdC}$ and 5-hydroxymethylcytosine (5hmdC) [71]. The latter, is also capable for detection of the unique phosphothioate modifications of the phospho-ribose backbone, found in some bacteria $[72,73]$. Expansion of these commercial platforms to high abundant epigenetic marks is the first step to enable them to detect any DNA modifications, including DNA adducts derived from endogenous or exogenous sources.

\subsubsection{Data analyses}

\subsubsection{ONT/ELIGOS.}

Our team developed the Epitranscriptional/ Epigenomical Landscape Inferring from Glitches of ONT Signals (ELIGOS) software that uses ONT data to simultaneously detect RNA and DNA modifications, including DNA adducts [55,74,75]. The ONT/ELIGOS platform is a powerful tool for (i) detecting DNA adducts and (ii) discriminating DNA adducts of different sizes, regiochemistry, and functional groups [55]. ELIGOS takes this error information from the standard base-calling algorithm and calculates the odds ratios at each site as an indicator for a potential DNA adduct (Figure 1)[55].

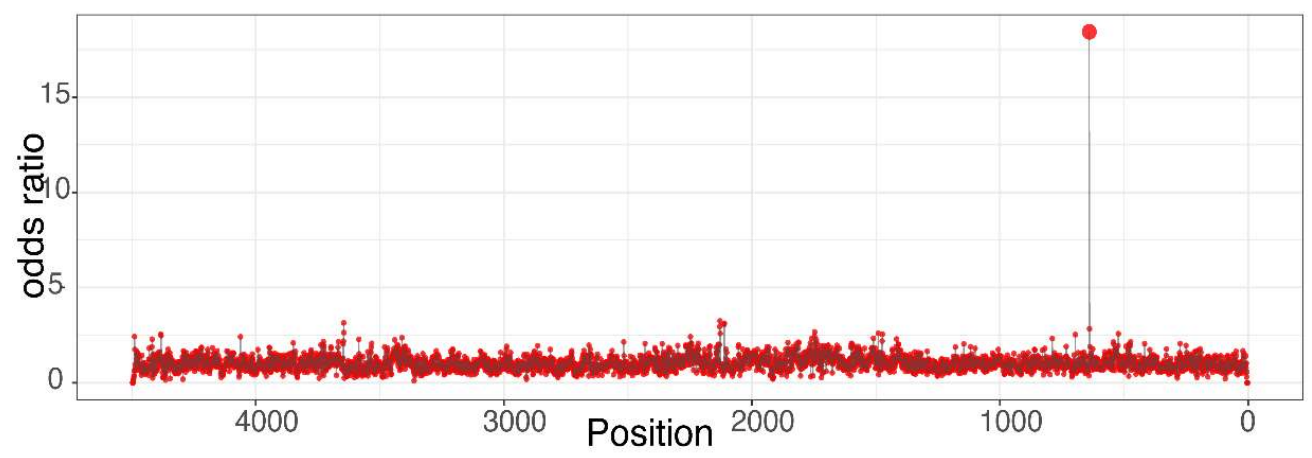

Figure 1. Representative Odds ratios plot of a plasmid carrying a DNA adduct at position G640.

Next, ELIGOS generates a radar plot displaying the multiplex disturbances of the raw ONT signal from the DNA adduct in relationship to the dG-containing control plasmid (Figure 2a)[55]. The radar plot shows an 11-base sequence that covers the DNA adduct along with 5 preceding and 5 trailing deoxynucleotides that dwell in the nanopore during sequencing $[55,76]$. These radar plots are characteristic for each DNA adduct at each given position. The radar plots are used to identify the type of DNA adduct by comparing them to plots of standard DNA that contains the DNA adduct at the same sequence position and within the same sequence context (Figure 2a). Disturbances in the raw ONT signal are used for multivariant statistical analyses to obtain a measure of separation, as shown in Figure 2b. 


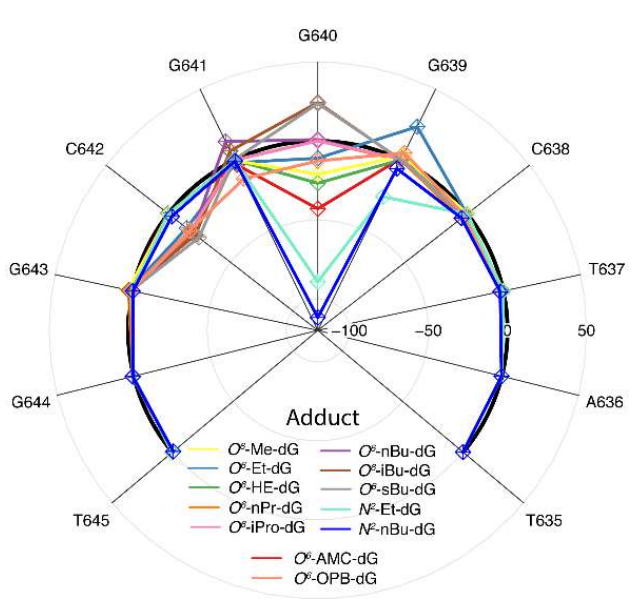

(a)

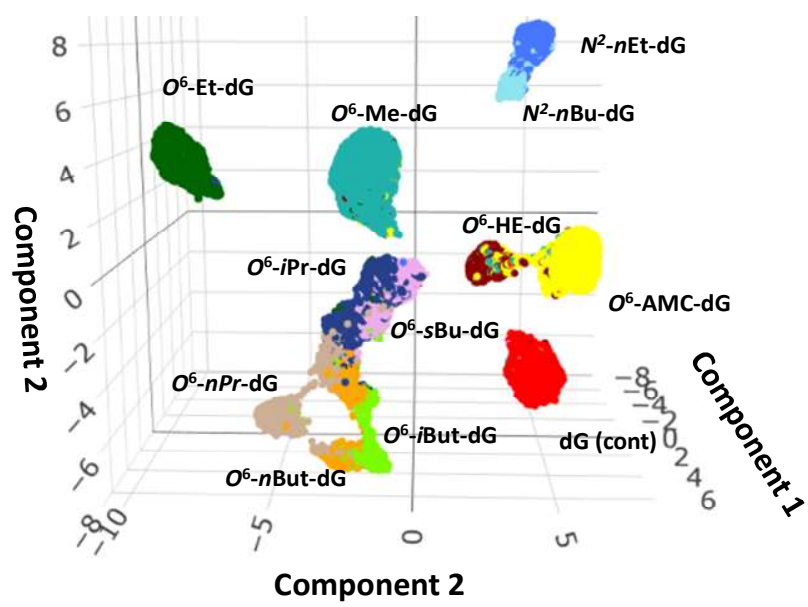

(b)

Figure 2. Characterization and separation of DNA adducts based on raw ONT signal: (a) Radar plot displaying the disturbance in raw ONT signal of 12 DNA adducts at position G640, compared to dG-containing control plasmid.; (b) Principal Component Analysis plot of ONT reads from 12 plasmids containing a site-specific DNA adduct or dG (red) at position G640. The separation is driven by the alkyl chain length and, to some extent, by the isomeric structures for the propyl- and butyl-adducts.

\subsubsection{CRISPR/cas-9 targeted sequencing.}

DNA adduct measurement by single molecule sequencing technologies will generate a huge amount of data because the DNA adduct levels are extremely low. In principle one would need to sequence the whole genome $10^{\wedge} 8$ times to get a DNA adduct level at each site of 1 adduct $/ 10^{\wedge} 8 \mathrm{nnt}$ as commonly reported. However, drawing from previous mapping approaches using CRISPR/cas-9 targeted sequencing will increase the number of reads and thereby sensitivity at the sites of interest such as cancer driver genes or mutation hotspots [77-79]. The CRISPR/cas-9 targeted sequencing may target DNA segments of 1,000 to 10,000 base pairs in length that can be read by ONT as a single molecule.

\subsubsection{Limit of Detection.}

The results of these single molecule analyses are number of DNA adduct $X$ at Site $Y$ per total number of DNA molecules / DNA molecules analyzed containing the site of interest, including unmodified DNA and DNA molecules carrying mutations or modification at different sites. In theory a DNA segment of 2000 base pairs length that has been read 100,000 times has a limit of detection of;

- 1 adduct at a given site/ per $10^{\wedge} 5$ unmodified bases at position $Y$

(e.g alkyl-dG/10^5 $\mathrm{dG}$ at position $\mathrm{Y}$ ),

- 5 adducts per $10^{\wedge} 9$ unmodified nucleotides

( e.g alkyl-dG/10^9 nnt),

- or approximately 1 adducts per $10^{\wedge} 9$ corresponding nucleotides

(e.g. alkyl-dG/10^9 dG).

This theoretical limits of detection of ONT/ELIGIOS are in the range of the levels reported for endogenous and exogenous DNA adducts.

\section{Discussion}

Below we highlight some selected studies showing the application and utility of DNA adduct research.

\subsection{DNA adduct levels.}


The first question in the DNA adduct field was, and still is, to demonstrate the formation of exposure induced DNA adducts. For example, Benzo(a)pyrene $(\mathrm{B}[a] \mathrm{P})$ a ubiquitous environmental and occupational carcinogen, and tobacco smoke constituent has been studied extensively. $\mathrm{B}[a] \mathrm{P}$ requires metabolic activation to the reactive 7,8,-diepoxy9,10-dihydroxy-benzo[a]pyrene (BPDE) that ultimately forms the promutagenic $N^{2}$ BPDE-dG adduct. Applying an ultra-sensitive LC-MS method, with a limit of detection of one $N^{2}$-BPDE-dG adduct per $10^{\wedge} 11$ nucleotides ( 1 adduct per 10 human cells) levels in

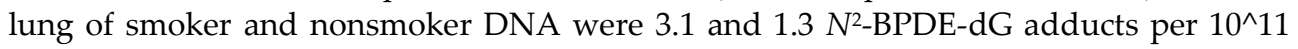
nucleotides, respectively [80].

With increases in sensitivity of the methodologies, it became apparent that DNA adducts are also formed by reactive compounds of endogenous origin, such as the 8-oxo-7,8dihydroguanine (8-oxo-dG). Oxidative modifications of DNA were first believed to be an artifact during sample handling and processing, which is in part still true, but their presence in all cellular DNA in nowadays well established [81,82]. Endogenous 8-oxo-dG in human peripheral blood lymphocytes measured by LC-MS are about one 8-oxo-dG per $10^{\wedge} 6 \mathrm{dG}[83,84]$. Consequently, endogenous 8-oxo-dG levels are five orders of magnitude higher than the exposure derived $N^{2}-B P D E-d G$.

Most studies are on base adducts, potentially disrupting the DNA base paring. However, adduct formation at the phosphor-ribose backbone has been proposed long ago and recently been shown for DNA-phosphate adducts formed by the tobacco-specific nitrosamine 4-(methylnitrosamino)-1-(3-pyridyl)-1-butanone [85].

\subsection{DNA adductomics.}

The emergence of nontargeted adductomics will generate a huge amount of DNA adduct data presumably representing the exposome. Studies of true DNA adductomics are limited, but first reports are very promising. For example, applying a nontargeted 'omics' approach, thousands of potential DNA adduct $m / z$ features were observed in human tissues such as lung, bronchia or saliva [86-88]. A challenging task will be to accurately identify the low abundant DNA adducts resulting from exogenous sources that are dwarfed by epigenic marks and endogenous DNA adducts, that are present at levels several orders of magnitude higher $[89,90]$.

\subsection{DNA adduct mapping.}

The second question in the DNA adduct field was, and still is, to determine the location of exposure-induced DNA adducts. Therefore, the mapping of B $[a] P$ derived DNA adducts has been reported genome wide [56] and for specific genes such as P53 [91], kRAS and $h R A S$ [92], suggesting DNA adduct formation at mutation hotspots in these genes. $\mathrm{B}[a] \mathrm{P}$ treatment however has been shown to also induce oxidative stress which his known to cause multiple DNA lesions recognized by the UvrABC Nuclease, potentially confounding the results [93]. Elegant stable isotope labeled experiments confirmed the preferred binding of BPDE to the mutation hotspot sequence motif in TP53 [94] settling this discussion.

Further, several approaches have been developed for genome wide mapping of 8oxo-dG with various degrees of resolution (reviewed by Poetsch [54]). The mapping revealed accumulation of 8-oxoG at sites of high nucleosome occupancy in yeast and different types of GC repeats accumulate large amounts of 8-oxoG, particularly telomeres and microsatellites [50], suggesting that DNA adduct formation is not random across the genome. These DNA adduct mapping studies highlight the importance of the DNA adduct location to elucidate subsequent biological outcomes.

The current nanopore-type Single Molecule DNA sequencing technologies do not reach the chemical specificity obtained by mass spectrometry, but efforts are underway to combine nanopore with mass spectrometry [95-97]. Further, since the ion signal disturbances are cause by physio-chemical properties of the DNA molecule transitioning the nanopore, 
refined artificial intelligence (AI) assisted data analyses in future may allow chemical specific identifications of the DNA adducts at any location in the genome. This novel technology is still in the implementation phase and future studies are needed to evaluate and establishing chemical specificity, sensitivity and accuracy for measuring DNA adducts.

\section{Conclusions}

With the current technologies on hand, it's easy to determine whether DNA adducts form in the target tissue to establish the internal dose derived from external exposure. When the goal is to understand the exposome, mass spectrometry-based adductomics is a method of choice. Detection and quantitation of DNA adducts derived from a mixture of pollutants in the target tissue will unambiguously demonstrate, with high chemical specificity, that the subject has been exposed and the toxicant has reached the tissue of concern.

If the goal is to understand how exposure leads to changes in cell homeostasis and promotes or prevents disease development many DNA adduct mapping approaches are available. These mapping methodologies will enable the investigator to demonstrate, with biological specificity, that the exposure or treatment induces modifications in the DNA at the promotor or gene region of interest.

The new Single Molecule DNA sequencing technologies are expected to be suitable for addressing both questions mentioned above. They will provide a comprehensive picture of the DNA adducts level and their locations across the genome.

Lastly, a novel approach is needed for meaningful interpretation of the DNA adductome-based exposomes and future DNA adduct maps to improve our understand in cancer etiology and explain the origin of mutational signatures established for various tumor types [98].

\section{Patents}

"Not applicable."

\section{Supplementary Materials:}

"Not applicable."

\section{Author Contributions:.}

This commentary was conceptualized by G.B.. The original draft was prepared by G.B and reviewed by I.N, who prepared the figures and visualizations.

\section{Funding:}

"Not applicable."

Institutional Review Board Statement:

"Not applicable."

Informed Consent Statement:

"Not applicable."

\section{Data Availability Statement:}

"Not applicable."

Acknowledgments: We are thankful to Dr David Ussery for careful review of the manuscripts and constructive comments.

\section{Conflicts of Interest:}

The authors declare no conflict of interest. 


\section{References}

1. Swenberg, J.A.; Lu, K.; Moeller, B.C.; Gao, L.; Upton, P.B.; Nakamura, J.; Starr, T.B. Endogenous versus Exogenous DNA Adducts: Their Role in Carcinogenesis, Epidemiology, and Risk Assessment. Toxicological Sciences 2011, 120, S130-S145, doi:10.1093/toxsci/kfq371.

2. Reiner, B.; Zamenhof, S. STUDIES ON THE CHEMICALLY REACTIVE GROUPS OF DEOXYRIBONUCLEIC ACIDS. Journal of Biological Chemistry 1957, 228, 475-486.

3. Hwa Yun, B.; Guo, J.; Bellamri, M.; Turesky, R.J. DNA Adducts: Formation, Biological Effects, and New Biospecimens for Mass Spectrometric Measurements in Humans. Mass spectrometry reviews 2020, 39, 55-82, doi:10.1002/mas.21570.

4. Tarun, M.; Rusling, J.F. Measuring DNA Nucleobase Adducts Using Neutral Hydrolysis and Liquid Chromatography-Mass Spectrometry. Critical reviews in eukaryotic gene expression 2005, 15, 295-316.

5. Boysen, G.; Hecht, S.S. Analysis of DNA and Protein Adducts of Benzo[a]Pyrene in Human Tissues Using Structure-Specific Methods. Mutation research 2003, 543, 17-30, doi:10.1016/s1383-5742(02)00068-6.

6. Boysen, G.; Pachkowski, B.F.; Nakamura, J.; Swenberg, J.A. The Formation and Biological Significance of N7-Guanine Adducts. Mutation research 2009, 678, 76-94, doi:10.1016/j.mrgentox.2009.05.006.

7. van Zeeland, A.A. Molecular Dosimetry of Chemical Mutagens. Relationship between DNA Adduct Formation and Genetic Changes Analyzed at the Molecular Level. Mutat.Res 1996, 353, 123-150.

8. de Bont, R.; van Larebeke, N. Endogenous DNA Damage in Humans: A Review of Quantitative Data. Mutagenesis 2004, 19, 169-185, doi:10.1093/mutage/geh025.

9. Rundle, A. Carcinogen-DNA Adducts as a Biomarker for Cancer Risk. Mutat.Res 2006, 600, 23-36.

10. Bartsch, H. DNA Adducts in Human Carcinogenesis: Etiological Relevance and Structure-Activity Relationship. Mutation Research 1996, 340, 67-79.

11. Harris, C.C. Chemical and Physical Carcinogenesis: Advances and Perspectives for the 1990s. Cancer Research $1991,51$.

12. Harvey, R.G. Historical Overview of Chemical Carcinogenesis. In Chemical Carcinogenesis; Penning, T.M., Ed.; Humana Press: Totowa, NJ, 2011; Vol. 6, pp. 1-26 ISBN 9781617379949.

13. Hartwig, A.; Arand, · Michael; Epe, · Bernd; Guth, S.; Jahnke, G.; Lampen, A.; Martus, · Hans-Jörg; Monien, · Bernhard; Rietjens, I.M.C.M.; Schmitz-Spanke, S.; et al. Mode of Action-Based Risk Assessment of Genotoxic Carcinogens. Archives of Toxicology 2020, 94, 1787-1877, doi:10.1007/s00204-020-02733-2.

14. La, D.K.; Swenberg, J.A. DNA Adducts: Biological Markers of Exposure and Potential Applications to Risk Assessment. Mutation Research 1996, 365, 129-146.

15. Phillips, D.H. DNA Adducts as Markers of Exposure and Risk. Mutation Research/Fundamental and Molecular Mechanisms of Mutagenesis 2005, 577, 284-292.

16. Poirier, M.C. DNA Adducts as Exposure Biomarkers and Indicators of Cancer Risk. Environ.Health Perspect. 1997, 105 Suppl, 907-912.

17. Pottenger, L.H.; Boysen, G.; Brown, K.; Cadet, J.; Fuchs, R.P.; Johnson, G.E.; Swenberg, J.A. Understanding the Importance of Low-Molecular Weight (Ethylene Oxide- and Propylene Oxide-Induced) DNA Adducts and Mutations in Risk Assessment: Insights from 15 Years of Research and Collaborative Discussions. Environmental and molecular mutagenesis 2019, 60, 100-121, doi:10.1002/em.22248.

18. Balbo, S.; Turesky, R.J.; Villalta, P.W. DNA Adductomics. Chemical research in toxicology 2014, 27, 356-366, doi:10.1021/tx4004352.

19. Walmsley, S.J.; Guo, J.; Wang, J.; Villalta, P.W.; Turesky, R.J. Methods and Challenges for Computational Data Analysis for DNA Adductomics. Chemical research in toxicology 2019, 32, 2156-2168, doi:10.1021/acs.chemrestox.9b00196.

20. Guo, J.; Turesky, R.J. Emerging Technologies in Mass Spectrometry-Based DNA Adductomics. High-Throughput $2019,8$. 
21. Alhegaili, A.S.; Ji, Y.; Sylvius, N.; Blades, M.J.; Karbaschi, M.; Tempest, H.G.; Jones, G.D.D.; Cooke, M.S. Genome-Wide Adductomics Analysis Reveals Heterogeneity in the Induction and Loss of Cyclobutane Thymine Dimers across Both the Nuclear and Mitochondrial Genomes. International journal of molecular sciences 2019, 20, doi:10.3390/ijms20205112.

22. Phillips, D.H.; Arlt, V.M. The 32p-Postlabeling Assay for Dna Adducts. Nature Protocols 2007, 2, 2772-2781, doi:10.1038/nprot.2007.394.

23. Cummings, J.; French, R.C.; Smyth, J.F. Application of High-Performance Liquid Chromatography for Recognition of Covalent Nucleic Acid Modification with Anticancer Drugs. Journal of chromatography 1993, 618, 251-276, doi:10.1016/03784347(93)80037-5.

24. Koc, H.; Swenberg, J.A. Applications of Mass Spectrometry for Quantitation of DNA Adducts. J Chromatogr B 2002, 778, 323343.

25. Farmer, P.B.; Singh, R. Use of DNA Adducts to Identify Human Health Risk from Exposure to Hazardous Environmental Pollutants: The Increasing Role of Mass Spectrometry in Assessing Biologically Effective Doses of Genotoxic Carcinogens. Mutation research 2008, 659, 68-76, doi:10.1016/J.MRREV.2008.03.006.

26. Dole, M.; Mack, L.L.; Hines, R.L.; Mobley, R.C.; Ferguson, L.D.; Alice, M.B. Molecular Beams of Macroions. The Journal of Chemical Physics 1968, 49, 2240-2249, doi:10.1063/1.1670391.

27. Fenn, J.B.; Mann, M.; Meng, C.K.; Wong, S.F.; Whitehouse, C.M. Electrospray Ionization-Principles and Practice. Mass Spectrometry Reviews 1990, 9, 37-70, doi:10.1002/mas.1280090103.

28. Guo, J.; Villalta, P.W.; Weight, C.J.; Bonala, R.; Johnson, F.; Rosenquist, T.A.; Turesky, R.J. Targeted and Untargeted Detection of DNA Adducts of Aromatic Amine Carcinogens in Human Bladder by Ultra-Performance Liquid Chromatography-HighResolution Mass Spectrometry. Chemical Research in Toxicology 2018, 31, doi:10.1021/acs.chemrestox.8b00268.

29. Guo, J.; J. Turesky, R.; Tarifa, A.; P. DeCaprio, A.; S. Cooke, M.; J. Walmsley, S.; W. Villalta, P. Development of a DNA Adductome Mass Spectral Database. Chemical Research in Toxicology 2020, 33, 852-854, doi:10.1021/acs.chemrestox.0c00031.

30. Behl, T.; Rachamalla, M.; Najda, A.; Sehgal, A.; Singh, S.; Sharma, N.; Bhatia, S.; Al-harrasi, A.; Chigurupati, S.; Vargas-de-lacruz, C.; et al. Applications of Adductomics in Chemically Induced Adverse Outcomes and Major Emphasis on DNA Adductomics: A Pathbreaking Tool in Biomedical Research. International journal of molecular sciences 2021, 22, doi:10.3390/IJMS221810141.

31. Olinski, R.; Rozalski, R.; Gackowski, D. Mass Spectrometry-Based Analysis of DNA Modifications: Potential Applications in Basic Research and Clinic. Methods in molecular biology (Clifton, N.J.) 2021, 2198, 27-35, doi:10.1007/978-1-0716-0876-0_3.

32. Ma, B.; Stepanov, I.; Hecht, S.S. Recent Studies on DNA Adducts Resulting from Human Exposure to Tobacco Smoke. Toxics 2019, Vol. 7, Page 16 2019, 7, 16, doi:10.3390/TOXICS7010016.

33. Monien, B.H. Mass Spectrometric DNA Adduct Quantification by Multiple Reaction Monitoring and Its Future Use for the Molecular Epidemiology of Cancer. Advances in experimental medicine and biology 2019, 1140, 743-751, doi:10.1007/978-3-03015950-4_44.

34. Tretyakova, N.; Goggin, M.; Sangaraju, D.; Janis, G. Quantitation of DNA Adducts by Stable Isotope Dilution Mass Spectrometry. Chemical research in toxicology 2012, 25, 2007-2035, doi:10.1021/TX3002548.

35. Zhang, P.; Carlsten, C.; Chaleckis, R.; Hanhineva, K.; Huang, M.; Isobe, T.; Koistinen, V.M.; Meister, I.; Papazian, S.; Sdougkou, K.; et al. Defining the Scope of Exposome Studies and Research Needs from a Multidisciplinary Perspective. Environmental Science and Technology Letters 2021, 8.

36. Murray, K.J.; Carlson, E.S.; Stornetta, A.; Balskus, E.P.; Villalta, P.W.; Balbo, S. Extension of Diagnostic Fragmentation Filtering for Automated Discovery in DNA Adductomics. Analytical Chemistry 2021, 93, doi:10.1021/acs.analchem.0c04895.

37. Htun, H.; Johnston, B.H. Mapping Adducts of DNA Structural Probes Using Transcription and Primer Extension Approaches. Methods in enzymology 1992, 212, 272-294, doi:10.1016/0076-6879(92)12017-k. 
38. Sinden, R.R.; Ussery, D.W. Analysis of DNA Structure in Vivo Using Psoralen Photobinding: Measurement of Supercoiling, Topological Domains, and DNA-Protein Interactions. Methods in enzymology 1992, 212, 319-335, doi:10.1016/00766879(92)12020-q.

39. Cao, B.; Wu, X.; Zhou, J.; Wu, H.; Liu, L.; Zhang, Q.; Demott, M.S.; Gu, C.; Wang, L.; You, D.; et al. Nick-Seq for SingleNucleotide Resolution Genomic Maps of DNA Modifications and Damage. Nucleic Acids Research 2020, 48, 6715-6725, doi:10.1093/NAR/GKAA473.

40. Denissenko, M.F.; Venkatachalam, S.; Ma, Y.H.; Wani, A.A. Site-Specific Induction and Repair of Benzo[a]Pyrene Diol Epoxide DNA Damage in Human H-Ras Protooncogene as Revealed by Restriction Cleavage Inhibition. Mutation research 1996, 363, 27-42, doi:10.1016/0921-8777(95)00059-3.

41. Denissenko, M.F.; Pao, A.; Tang, M. -s.; Pfeifer, G.P. Preferential Formation of Benzo[a]Pyrene Adducts at Lung Cancer Mutational Hotspots in P53. Science (New York, N.Y.) 1996, 274, 430-432, doi:10.1126/science.274.5286.430.

42. Denissenko, M.F.; Chen, J.X.; Tang, M.S.; Pfeifer, G.P. Cytosine Methylation Determines Hot Spots of DNA Damage in the Human P53 Gene. Proceedings of the National Academy of Sciences of the United States of America 1997, 94, 3893-3898, doi:10.1073/pnas.94.8.3893.

43. Mao, P.; Smerdon, M.J.; Roberts, S.A.; Wyrick, J.J. Chromosomal Landscape of UV Damage Formation and Repair at SingleNucleotide Resolution. Proceedings of the National Academy of Sciences of the United States of America 2016, 113, 9057-9062, doi:10.1073/pnas.1606667113.

44. Mao, P.; Brown, A.J.; Malc, E.P.; Mieczkowski, P.A.; Smerdon, M.J.; Roberts, S.A.; Wyrick, J.J. Genome-Wide Maps of Alkylation Damage, Repair, and Mutagenesis in Yeast Reveal Mechanisms of Mutational Heterogeneity. Genome research 2017, 27, 1674-1684, doi:10.1101/gr.225771.117.

45. Hu, J.; Li, W.; Adebali, O.; Yang, Y.; Oztas, O.; Selby, C.P.; Sancar, A. Genome-Wide Mapping of Nucleotide Excision Repair with XR-Seq. Nature Protocols 2019, 14, 248-282, doi:10.1038/s41596-018-0093-7.

46. Li, W.; Hu, J.; Adebali, O.; Adar, S.; Yang, Y.; Chiou, Y.-Y.; Sancar, A. Human Genome-Wide Repair Map of DNA Damage Caused by the Cigarette Smoke Carcinogen Benzo[a]Pyrene. Proceedings of the National Academy of Sciences of the United States of America 2017, 114, 6752-6757, doi:10.1073/pnas.1706021114.

47. Govindan, R.; Szczesna, A.; Ahn, M.; Schneider, C.; Gonzalez Mella, P.F.; Barlesi, F.; Han, B.; Ganea, D.E.; von Pawel, J.; Vladimirov, V.; et al. Phase III Trial of Ipilimumab Combined With Paclitaxel and Carboplatin in Advanced Squamous NonSmall-Cell Lung Cancer. Journal of clinical oncology : official journal of the American Society of Clinical Oncology 2017, 35, 34493457, doi:10.1200/JCO.2016.71.7629.

48. Yang, Y.; Hu, J.; Selby, C.P.; Li, W.; Yimit, A.; Jiang, Y.; Sancar, A. Single-Nucleotide Resolution Analysis of Nucleotide Excision Repair of Ribosomal DNA in Humans and Mice. The Journal of biological chemistry 2019, 294, 210-217, doi:10.1074/jbc.RA118.006121.

49. Shu, X.; Xiong, X.; Song, J.; He, C.; Yi, C. Base-Resolution Analysis of Cisplatin-DNA Adducts at the Genome Scale. Angewandte Chemie (International ed. in English) 2016, 55, 14246-14249, doi:10.1002/anie.201607380.

50. Wu, J.; McKeague, M.; J. Sturla, S. Nucleotide-Resolution Genome-Wide Mapping of Oxidative DNA Damage by Click-CodeSeq. Journal of the American Chemical Society 2018, 140, 9783-9787, doi:10.1021/jacs.8b03715.

51. Schutsky, E.K.; Denizio, J.E.; Hu, P.; Liu, M.Y.; Nabel, C.S.; Fabyanic, E.B.; Hwang, Y.; Bushman, F.D.; Wu, H.; Kohli, R.M. Nondestructive, Base-Resolution Sequencing of 5-Hydroxymethylcytosine Using a DNA Deaminase. Nature Biotechnology 2018, 36, 1083-1090, doi:10.1038/nbt.4204.

52. Vitelli, V.; Galbiati, A.; Iannelli, F.; Pessina, F.; Sharma, S.; d'Adda di Fagagna, F. Recent Advancements in DNA DamageTranscription Crosstalk and High-Resolution Mapping of DNA Breaks. Annual review of genomics and human genetics 2017, 18, 87-113, doi:10.1146/annurev-genom-091416-035314. 
53. Amente, S.; Scala, G.; Majello, B.; Azmoun, S.; Tempest, H.G.; Premi, S.; Cooke, M.S. Genome-Wide Mapping of Genomic DNA Damage: Methods and Implications. Cellular and Molecular Life Sciences 2021, 78.

54. Poetsch, A.R. The Genomics of Oxidative DNA Damage, Repair, and Resulting Mutagenesis. Computational and structural biotechnology journal 2020, 18, 207-219, doi:10.1016/j.csbj.2019.12.013.

55. Nookaew, I.; Jenjaroenpun, P.; Du, H.; Wang, P.; Wu, J.; Wongsurawat, T.; Moon, S.H.; Huang, E.; Wang, Y.; Boysen, G. Detection and Discrimination of DNA Adducts Differing in Size, Regiochemistry, and Functional Group by Nanopore Sequencing. Chemical research in toxicology 2020, 33, 2944-2952, doi:10.1021/acs.chemrestox.0c00202.

56. Perera, R.T.; Fleming, A.M.; Johnson, R.P.; Burrows, C.J.; White, H.S. Detection of Benzo[a]Pyrene-Guanine Adducts in Single-Stranded DNA Using the $\alpha$-Hemolysin Nanopore. Nanotechnology 2015, 26, 1-7, doi:10.1088/0957-4484/26/7/074002.

57. An, N.; Fleming, A.M.; White, H.S.; Burrows, C.J. Crown Ether-Electrolyte Interactions Permit Nanopore Detection of Individual DNA Abasic Sites in Single Molecules. Proceedings of the National Academy of Sciences of the United States of America 2012, 109, 11504-11509, doi:10.1073/pnas.1201669109/-/DCSupplemental.

58. An, N.; Fleming, A.M.; Rosecrans, N.C.; Liao, Y.; Burrows, C.J. Synthesis of Site-Specific Crown Ether Adducts to DNA Abasic Sites: 8-Oxo-7,8-Dihydro-2'-Deoxyguanosine and 2'-Deoxycytidine. Methods in molecular biology (Clifton, N.J.) 2019, 1973, 1525, doi:10.1007/978-1-4939-9216-4_2.

59. Liu, Z.J.; Martínez Cuesta, S.; van Delft, P.; Balasubramanian, S. Sequencing Abasic Sites in DNA at Single-Nucleotide Resolution. Nature Chemistry 2019, 11, 629-637, doi:10.1038/s41557-019-0279-9.

60. Tan, C.S.; Fleming, A.M.; Ren, H.; Burrows, C.J.; White, H.S. $\gamma$-Hemolysin Nanopore Is Sensitive to Guanine-to-Inosine Substitutions in Double-Stranded DNA at the Single-Molecule Level. Journal of the American Chemical Society 2018, 140, 1422414234, doi:10.1021/jacs.8b08153.

61. Zeng, T.; Fleming, A.M.; Ding, Y.; Ren, H.; White, H.S.; Burrows, C.J. Nanopore Analysis of the 5-Guanidinohydantoin to Iminoallantoin Isomerization in Duplex DNA. The Journal of organic chemistry 2018, 83, 3973-3978, doi:10.1021/acs.joc.8b00317.

62. Fleming, A.M.; Ding, Y.; Burrows, C.J. Oxidative DNA Damage Is Epigenetic by Regulating Gene Transcription via Base Excision Repair. Proceedings of the National Academy of Sciences 2017, 114, 2604-2609, doi:10.1073/pnas.1619809114.

63. Ding, Y.; Fleming, A.M.; Burrows, C.J. Sequencing the Mouse Genome for the Oxidatively Modified Base 8-Oxo-7,8Dihydroguanine by OG-Seq. Journal of the American Chemical Society 2017, 139, 2569-2572, doi:10.1021/jacs.6b12604.

64. Payne, A.; Holmes, N.; Rakyan, V.; Loose, M. BulkVis: A Graphical Viewer for Oxford Nanopore Bulk FAST5 Files. Bioinformatics 2019, 35, 2193-2198, doi:10.1093/bioinformatics/bty841.

65. Tyson, J.R.; O’Neil, N.J.; Jain, M.; Olsen, H.E.; Hieter, P.; Snutch, T.P. MinION-Based Long-Read Sequencing and Assembly Extends the Caenorhabditis Elegans Reference Genome. Genome research 2018, 28, 266-274, doi:10.1101/gr.221184.117.

66. Xu, L.; Seki, M. Recent Advances in the Detection of Base Modifications Using the Nanopore Sequencer. Journal of Human Genetics 2020, 65, 25-33, doi:10.1038/s10038-019-0679-0.

67. Rand, A.C.; Jain, M.; Eizenga, J.M.; Musselman-Brown, A.; Olsen, H.E.; Akeson, M.; Paten, B. Mapping DNA Methylation with High-Throughput Nanopore Sequencing. Nature methods 2017, 14, 411-413, doi:10.1038/nmeth.4189.

68. Simpson, J.T.; Workman, R.E.; Zuzarte, P.C.; David, M.; Dursi, L.J.; Timp, W. Detecting DNA Cytosine Methylation Using Nanopore Sequencing. Nature methods 2017, 14, 407-410, doi:10.1038/nmeth.4184.

69. Liu, Q.; Fang, L.; Yu, G.; Wang, D.; Xiao, C.-L.; Wang, K. Detection of DNA Base Modifications by Deep Recurrent Neural Network on Oxford Nanopore Sequencing Data. Nature communications 2019, 10, 2449, doi:10.1038/s41467-019-10168-2.

70. Ni, P.; Huang, N.; Zhang, Z.; Wang, D.-P.; Liang, F.; Miao, Y.; Xiao, C.-L.; Luo, F.; Wang, J. DeepSignal: Detecting DNA Methylation State from Nanopore Sequencing Reads Using Deep-Learning. Bioinformatics (Oxford, England) 2019, 35, 45864595, doi:10.1093/bioinformatics/btz276. 
71. Flusberg, B.A.; Webster, D.R.; Lee, J.H.; Travers, K.J.; Olivares, E.C.; Clark, T.A.; Korlach, J.; Turner, S.W. Direct Detection of DNA Methylation during Single-Molecule, Real-Time Sequencing. Nature methods 2010, 7, 461-465, doi:10.1038/nmeth.1459.

72. Cao, B.; Chen, C.; DeMott, M.S.; Cheng, Q.; Clark, T.A.; Xiong, X.; Zheng, X.; Butty, V.; Levine, S.S.; Yuan, G.; et al. Genomic Mapping of Phosphorothioates Reveals Partial Modification of Short Consensus Sequences. Nature Communications 2014, 5 , 3951, doi:10.1038/ncomms4951.

73. Ahlgren, N.A.; Chen, Y.; Needham, D.M.; Parada, A.E.; Sachdeva, R.; Trinh, V.; Chen, T.; Fuhrman, J.A. Genome and Epigenome of a Novel Marine Thaumarchaeota Strain Suggest Viral Infection, Phosphorothioation DNA Modification and Multiple Restriction Systems. Environmental Microbiology 2017, 19, 2434-2452, doi:10.1111/1462-2920.13768.

74. Jenjaroenpun, P.; Wongsurawat, T.; Pereira, R.; Patumcharoenpol, P.; Ussery, D.W.; Nielsen, J.; Nookaew, I. Complete Genomic and Transcriptional Landscape Analysis Using Third-Generation Sequencing: A Case Study of Saccharomyces Cerevisiae CEN.PK113-7D. Nucleic acids research 2018, 46, e38, doi:10.1093/nar/gky014.

75. Wongsurawat, T.; Jenjaroenpun, P.; Taylor, M.K.; Lee, J.; Tolardo, A.L.; Parvathareddy, J.; Kandel, S.; Wadley, T.D.; Kaewnapan, B.; Athipanyasilp, N.; et al. Rapid Sequencing of Multiple RNA Viruses in Their Native Form. Frontiers in Microbiology 2019, 10, 260, doi:10.3389/fmicb.2019.00260.

76. Wick, R.R.; Judd, L.M.; Holt, K.E. Performance of Neural Network Basecalling Tools for Oxford Nanopore Sequencing. Genome biology 2019, 20, 129, doi:10.1186/s13059-019-1727-y.

77. Gilpatrick, T.; Lee, I.; Graham, J.E.; Raimondeau, E.; Bowen, R.; Heron, A.; Downs, B.; Sukumar, S.; Sedlazeck, F.J.; Timp, W. Targeted Nanopore Sequencing with Cas9-Guided Adapter Ligation. Nature biotechnology 2020, 38, 433-438, doi:10.1038/s41587-020-0407-5.

78. Veres, A.; Gosis, B.S.; Ding, Q.; Collins, R.; Ragavendran, A.; Brand, H.; Erdin, S.; Cowan, C.A.; Talkowski, M.E.; Musunuru, K. Low Incidence of Off-Target Mutations in Individual CRISPR-Cas9 and TALEN Targeted Human Stem Cell Clones Detected by Whole-Genome Sequencing. Cell stem cell 2014, 15, 27-30, doi:10.1016/j.stem.2014.04.020.

79. Wongsurawat, T.; Jenjaroenpun, P.; de Loose, A.; Alkam, D.; Ussery, D.W.; Nookaew, I.; Leung, Y.K.; Ho, S.M.; Day, J.D.; Rodriguez, A. A Novel Cas9-Targeted Long-Read Assay for Simultaneous Detection of IDH1/2 Mutations and Clinically Relevant MGMT Methylation in Fresh Biopsies of Diffuse Glioma. Acta Neuropathologica Communications 2020, 8, 1-13, doi:10.1186/S40478-020-00963-0/FIGURES/4.

80. Villalta, P.W.; Hochalter, J.B.; Hecht, S.S. Ultrasensitive High-Resolution Mass Spectrometric Analysis of a DNA Adduct of the Carcinogen Benzo[a]Pyrene in Human Lung. Analytical chemistry 2017, 89, 12735-12742, doi:10.1021/acs.analchem.7b02856.

81. Collins, A.R.; Cadet, J.; Moller, L.; Poulsen, H.E.; Viña, J. Are We Sure We Know How to Measure 8-Oxo-7,8-Dihydroguanine in DNA from Human Cells? Archives of biochemistry and biophysics 2004, 423, 57-65, doi:10.1016/J.ABB.2003.12.022.

82. Collins, A.; Gedik, C.; Vaughan, N.; Wood, S.; White, A.; Dubois, J.; Rees, J.F.; Loft, S.; Møller, P.; Poulsen, H.; et al. Measurement of DNA Oxidation in Human Cells by Chromatographic and Enzymic Methods. Free radical biology E medicine 2003, 34, 1089-1099, doi:10.1016/S0891-5849(03)00041-8.

83. Boysen, G.; Collins, L.B.; Liao, S.; Luke, A.M.; Pachkowski, B.F.; Watters, J.L.; Swenberg, J.A. Analysis of 8-Oxo-7,8-Dihydro2'-Deoxyguanosine by Ultra High Pressure Liquid Chromatography-Heat Assisted Electrospray Ionization-Tandem Mass Spectrometry. Journal of chromatography. B, Analytical technologies in the biomedical and life sciences 2010, 878, 375-380, doi:10.1016/j.jchromb.2009.12.004.

84. Watters, J.J.L.; Satia, J.J. a; da Costa, K.-A.; Boysen, G.; Collins, L.B.; Morrow, J.D.; Milne, G.L.; Swenberg, J. a; Costa, K. Comparison of Three Oxidative Stress Biomarkers in a Sample of Healthy Adults. Biomarkers 2009, 14, 587-595, doi:10.3109/13547500903183954. 
85. Ma, B.; Zarth, A.T.; Carlson, E.S.; Villalta, P.W.; Upadhyaya, P.; Stepanov, I.; Hecht, S.S. Identification of More than 100 Structurally Unique DNA-Phosphate Adducts Formed during Rat Lung Carcinogenesis by the Tobacco-Specific Nitrosamine 4-(Methylnitrosamino)-1-(3-Pyridyl)-1-Butanone. Carcinogenesis 2018, 39, 232-241, doi:10.1093/carcin/bgx135.

86. Kanaly, R.A.; Matsui, S.; Hanaoka, T.; Matsuda, T. Application of the Adductome Approach to Assess Intertissue DNA Damage Variations in Human Lung and Esophagus. Mutation Research/Fundamental and Molecular Mechanisms of Mutagenesis 2007, 625, 83-93, doi:10.1016/J.MRFMMM.2007.05.006.

87. Bessette, E.E.; Goodenough, A.K.; Langouët, S.; Yasa, I.; Kozekov, I.D.; Spivack, S.D.; Turesky, R.J. Screening for DNA Adducts by Data-Dependent Constant Neutral Loss-Triple Stage Mass Spectrometry with a Linear Quadrupole Ion Trap Mass Spectrometer. Analytical Chemistry 2009, 81, 809-819, doi:10.1021/ac802096p.

88. Bessette, E.E.; Spivack, S.D.; Goodenough, A.K.; Wang, T.; Pinto, S.; Kadlubar, F.F.; Turesky, R.J. Identification of Carcinogen DNA Adducts in Human Saliva by Linear Quadrupole Ion Trap/Multistage Tandem Mass Spectrometry. Chemical Research in Toxicology 2010, 23, 1234-1244, doi:10.1021/tx100098f.

89. Iwashita, Y.; Ohnishi, I.; Matsushita, Y.; Ohtsuka, S.; Yamashita, T.; Inaba, K.; Fukazawa, A.; Ochiai, H.; Matsumoto, K.; Kurono, N.; et al. Geospatial Assessments of DNA Adducts in the Human Stomach: A Model of Field Cancerization. Cancers 2021, 13, 3728, doi:10.3390/cancers13153728.

90. Ohnishi, I.; Iwashita, Y.; Matsushita, Y.; Ohtsuka, S.; Yamashita, T.; Inaba, K.; Fukazawa, A.; Ochiai, H.; Matsumoto, K.; Kurono, N.; et al. Mass Spectrometric Profiling of DNA Adducts in the Human Stomach Associated with Damage from Environmental Factors. Genes and environment: the official journal of the Japanese Environmental Mutagen Society 2021, 43, 12, doi:10.1186/s41021-021-00186-2.

91. Tang, M.S.; Zheng, J.B.; Denissenko, M.F.; Pfeifer, G.P.; Zheng, Y. Use of UvrABC Nuclease to Quantify Benzo[a]Pyrene Diol Epoxide-DNA Adduct Formation at Methylated versus Unmethylated CpG Sites in the P53 Gene. Carcinogenesis 1999, 20, 1085-1089, doi:10.1093/carcin/20.6.1085.

92. Dittrich, K.A.; Krugh, T.R. Mapping of (+/-)-Anti-Benzo[a]Pyrene Diol Epoxide Adducts to Human c-Ha-Ras1 Protooncogene. Chemical research in toxicology 1991, 4, 277-281, doi:10.1021/TX00021A003.

93. Briedé, J.J.; Godschalk, R.W.L.; Emans, M.T.G.; de Kok, T.M.C.M.; van Agen, E.; van Maanen, J.; van Schooten, F.-J.; Kleinjans, J.C.S. In Vitro and in Vivo Studies on Oxygen Free Radical and DNA Adduct Formation in Rat Lung and Liver during Benzo[a]Pyrene Metabolism. Free radical research 2004, 38, 995-1002, doi:10.1080/10715760400000976.

94. Matter, B.; Wang, G.; Jones, R.; Tretyakova, N. Formation of Diastereomeric Benzo[a]Pyrene Diol Epoxide-Guanine Adducts in P53 Gene-Derived DNA Sequences. Chemical research in toxicology 2004, 17, 731-741, doi:10.1021/TX049974L.

95. Bush, J.; Maulbetsch, W.; Lepoitevin, M.; Wiener, B.; Mihovilovic Skanata, M.; Moon, W.; Pruitt, C.; Stein, D. The Nanopore Mass Spectrometer. The Review of scientific instruments 2017, 88, doi:10.1063/1.4986043.

96. Maulbetsch, W.; Wiener, B.; Poole, W.; Bush, J.; Stein, D. Preserving the Sequence of a Biopolymer's Monomers as They Enter an Electrospray Mass Spectrometer. Physical Review Applied 2016, 6, 054006, doi:10.1103/PHYSREVAPPLIED.6.054006/FIGURES/3/MEDIUM.

97. Brodbelt, J.S. Photodissociation Mass Spectrometry: New Tools for Characterization of Biological Molecules. Chemical Society reviews 2014, 43, 2757-2783, doi:10.1039/C3CS60444F.

98. Totsuka, Y.; Watanabe, M.; Lin, Y. New Horizons of DNA Adductome for Exploring Environmental Causes of Cancer. Cancer science 2021, 112, 7-15, doi:10.1111/cas.14666. 